Geophysical Research Abstracts, Vol. 7, 07124, 2005

SRef-ID: 1607-7962/gra/EGU05-A-07124

(C) European Geosciences Union 2005

\title{
Rainfall stochastic modeling for runoff forecasting
}

F. Russo (1), F. Lombardo (1), F. Napolitano (1) and E. Gorgucci (2)

(1) Department of Hydraulic, Transportations and Roads, University of Rome "La Sapienza", Rome, Italy, (2) Institute of Atmospheric Sciences and Climate, National Research Council, Rome, Italy (francesco.napolitano@ uniroma1.it / Fax +39 06-44585065)

Rainfall fields estimation over a catchment area is an important stage in many hydrological application. In this context weather radars have several advantages since a single site is able to obtain coverage over a vast area with very high temporal and spatial resolution and the advent of weather radar systems with dual polarization capability allowed progress on radar rainfall estimation and its hydro-meteorological applications. For these applications of radar data it was necessary to remove the ground clutter contamination with an algorithm based on the backscattering signal variance of the differential reflectivity. The calibration of the GDSTM model (Gaussian Displacements Spatial-Temporal Model), a cluster stochastic generation model in continuous space and time, is presented. In this model storms arrive in a Poisson process in time with cells occurring in each storm that cluster in space and time. The model is calibrated, using data collected by the weather radar Polar 55C located in Rome, inside a square area of $132 \times 132 \mathrm{~km}^{2}$, with the radar at the center.

The GDSTM is fitted to sequences of radar images with a time interval between the PPIs scans of 5 minutes. A generalized method of moments procedure is used for parameters estimation. For the validation of the ability of the model to reproduce the internal structure of rain event a geomorphological rainfall-runoff model based on width function (WFIUH) was calibrated using data simulated and observed data.

Several rainfall fields are generated with the stochastic model and then they are used as input of the WFIUH model so that the forecasted discharges can be compared to the observed ones. 